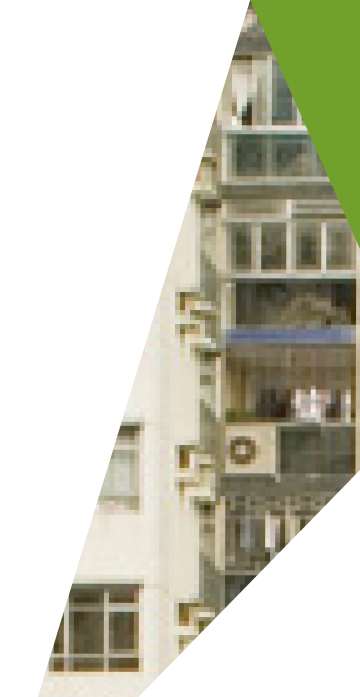

A RTE Y SALUD

\title{
ARTE Y BIENESTAR EMOCIONAL EN TIEMPOS DE CONFINAMIENTO OBLIGATORIO:
} EVIDENCIAS PARA ESTRECHAR LA RELACIÓN ENTRE LAS POLÍTICAS CULTURALES Y DE SALUD MENTAL

ART AND EMOTIONAL WELL-BEING IN TIMES OF MANDATORY CONFINEMENT:

EVIDENCE TO STRENGTHEN THE RELATIONSHIP BETWEEN CULTURAL AND MENTAL HEALTH POLICIES

TELMA BARRANTES FERNÁNDEZ ESTEBAN CRUZ HIDALGO JOSÉ FRANCISCO RANGEL PRECIADO FRANCISCO MANUEL PAREJO MORUNO

Universidad de Extremadura 


\section{RESUMEN}

La pandemia del coronavirus ha obligado a los gobiernos de todo el mundo a promulgar medidas desconocidas hasta ahora para frenar sus peores consecuencias económicas y sociales. En España, el Real Decreto 463/2020 de 14 de marzo de 2020 declaró el estado de alarma para la gestión de la situación de crisis sanitaria ocasionada por la COVID-19. Desde ese momento se procedió al confinamiento generalizado de la población en sus casas, permaneciendo los menores de edad encerrados entre el 14 de marzo y el 26 de abril de 2020. En este estudio identificamos los comportamientos, emociones y síntomas físicos que han podido padecer los menores derivados del confinamiento. También analizamos si el desarrollo de actividades creativas durante el confinamiento ha motivado en ellos alteraciones emocionales y conductuales. Los resultados constatan que la realización de estas actividades tiene efectos positivos, contribuyendo a mitigar de forma significativa algunos síntomas como el nerviosismo o el apego excesivo a los adultos, mejorando también de forma perceptible su conducta y actitud ante esta situación extraordinaria. La investigación se completa comparando los efectos del confinamiento con la hospitalización pediátrica, incidiendo en los cambios actitudinales y de sociabilidad de los menores. Frente a la tendencia actual hacia el desplazamiento de la educación artística de los planes de estudios, las evidencias encontradas refuerzan las demandas de que el arte ocupe un lugar esencial en la sociedad; un papel que va más allá del estrecho espacio tradicionalmente acotado a la Economía de la Cultura y dirigido por las actividades de mercado. Como conclusión, reflexionamos sobre la dirección que la política económica debería tener para poner en relación la política cultural y las políticas de salud mental desde etapas tempranas.

\section{PALABRAS CLAVE}

Aislamiento social, Educación artística, Beneficios emocionales del arte, Política social,

Bienestar en menores, Desmercantilización de la cultura

\section{A B S T R ACT}

The coronavirus pandemic has forced governments around the world to enact hitherto unknown measures to curb its worst economic and social consequences. In Spain, Royal Decree 463/2020 of March 14, 2020 declares the state of alarm for the management of the health crisis situation caused by COVID-19. From that moment, the population was confined to their homes in a general way, with minors being locked up between March 14 and April 26, 2020. In this study we identify the behaviors, emotions and physical symptoms that they may have suffered minors derived from confinement. We also analyze whether the development of creative activities during confinement has motivated emotional and behavioral changes in them. The results confirm that the performance of these activities has positive effects, contributing to significantly mitigate some symptoms such as nervousness or excessive attachment to adults, also significantly improving their behavior and attitude in this extraordinary situation. The investigation is completed comparing the effects of confinement with pediatric hospitalization, influencing the attitudinal and sociability changes of minors. Faced with the current trend towards the displacement of artistic education from the curricula, the evidence found reinforces the demands that art occupy a central place in society; a role that goes beyond the narrow space traditionally limited to the Economy of Culture and directed by the market. In conclusion, we reflect on the direction that economic policies should have to relate cultural policy and mental health policies from the early stages.

\section{KEYWORDS}

Social isolation, Artistic education, Emotional benefits of art; Social policy, Well-being in minors, De-commodification of culture. 


\section{INTRODUCCIÓN}

Con la aparición del coronavirus SARS-CoV-2 la cotidianidad en la vida de los individuos se ha visto alterada. La epidemia ha obligado a modificar cada uno de los comportamientos sociales, laborales y de ocio, así como las actuaciones en los colegios y universidades, e incluso en los parques y supermercados. En definitiva, la nueva normalidad sobrevenida por la enfermedad infecciosa COVID-19 ha traído consigo una nueva sociabilidad.

En otro orden de cosas, la creatividad constituye una herramienta integral del desarrollo emocional y educativo en los menores, que favorece el autodescubrimiento y potencia las capacidades subyacentes, fomentando también el afrontamiento de situaciones emocionalmente desestabilizadoras (Monroy, 2006; Carpena y López, 2012). Con el confinamiento, el aislamiento social ha paralizado las interacciones sociales directas de los menores con otros menores. La paralización del contacto social, tanto en los espacios de enseñanza como en los entornos lúdicos donde tienden a relacionarse, exige de preguntarse sobre cómo están afectando estas circunstancias a los menores, algo que hemos hecho en este estudio que centramos en las semanas donde el distanciamiento social ha sido total.

Por un lado, es conocido que el aislamiento social produce un impacto de diversa índole en los individuos. Y que son las interacciones y relaciones sociales que el individuo mantiene las que refuerzan el afrontamiento de situaciones traumáticas, al reducir su inseguridad y estrés, y al minimizar aquellos riesgos sobre los cuales se siente vulnerable (Mayo, 2018, p.106). Estimamos que, a lo largo del confinamiento, esta vulnerabilidad se ha visto fuer- temente desatendida en los menores, ya que el contacto social durante varias semanas no ha sido posible por causa de las estrictas y necesarias medidas aplicadas para proteger la salud pública. Por otro lado, observando la explosión de iniciativas artísticas que han surgido en pleno confinamiento, nos preguntamos si estas actividades creativas podían estar aliviando o conteniendo dichos síntomas derivados del aislamiento social. En este sentido, se percibe que la creatividad ha podido ser un puente social frente a la situación adversa que nos ha sobrevenido, al dotar a los individuos de recursos para mejorar su resiliencia y su capacidad de adaptación a esta situación traumática. En suma, el arte se ha reivindicado como un acto individual y colectivo al mismo tiempo, cumpliendo un objetivo fundamental de socialización (Fernández-Cao, 2011 , p.33), tan necesario en este tiempo de confinamiento, verificándose su doble carácter "autónomo" y de "fait social" que le atribuyera el filósofo Adorno cinco décadas atrás (Adorno, 1970 [1983], pp. 15 y 23).

Bajo este planteamiento, nuestra hipótesis de partida suscita que el arte es un acto recurrente que alivia las consecuencias negativas que derivan de la falta de contacto social, tan necesaria siempre y más en situaciones como la que estamos viviendo. Para contrastarla hemos construido un cuestionario que permite recoger los síntomas derivados por la situación extraordinaria de aislamiento social que los menores sufren, así como la realización de actividades artísticas durante el confinamiento, siendo sensible también al comportamiento observado por los padres en sus hijos durante las semanas de cuarentena. El objetivo de nuestro estudio es analizar si se evidencia una relación 
positiva entre el desarrollo de estas actividades y el estado emocional de los menores, en el contexto de una situación traumática que provoca grandes cambios en sus vidas. Como no existen en la literatura estudios sobre experiencias similares, dada la novedad histórica de una pandemia global en tiempos modernos, creemos conveniente comparar nuestros resultados con los examinados en la literatura sobre hospitalización pediátrica (Del Barrio y Mestre, 1989; Lizasoáin y Polaino-Lorente, 1992; Fernández-Castillo y López-Naranjo, 2006; Hernández y Rabadán 2013; Cruz, Mejías y Machado, 2014). Nuestros resultados indican que los efectos asociados al aislamiento social derivado de la pandemia guardan cierta semejanza con los encontrados en tales circunstancias, tanto en aspectos emocionales como cognitivo-conductuales.

El artículo se estructura de la siguiente forma. Tras este primer apartado a modo de introducción, abordamos en un segundo punto el contexto inusual que caracteriza el momento en el que transcurre nuestra investigación: la pandemia que ha obligado al mundo a encerrarse en sus casas durante semanas en pleno siglo XXI. También aquí revisamos la literatura sobre hospitalización pediátrica, que nos sirve de base sobre la cual construir nuestra investigación ante la carencia de antecedentes concretos de esta situación. En el tercer punto se expone el papel jugado por el arte y la creatividad en diversas dimensiones vitales para el individuo. En el cuarto apartado desarrollamos la metodología utilizada, las características de la muestra y los resultados obtenidos sobre los beneficios declarados por los padres de los menores con relación a la realización de actividades creativas durante el periodo de encierro estricto. Por último, y a modo de conclusiones, comparamos nuestros resultados con aquellos observados en la literatura sobre hospitalización pediátrica y realizamos una reflexión sobre los beneficios del arte para los individuos.

\section{EFECTOS DEL AISLAMIENTO SOCIAL EN MENORES}

Ciertamente, por qué negarlo, no sabemos mucho de la enfermedad infecciosa $\mathrm{CO}$ VID-19. Su origen sigue siendo controvertido y borroso. Los estudios sobre su diseminación por el mundo ofrecen información que cambia los consensos frágiles previos; y mientras el virus conviva con nosotros, el mundo estará sujeto a una incertidumbre gobernada por su amenaza. Sea lo que sea, la normalidad se modifica y toma cuerpo mientras redactamos este estudio. Y tardará un tiempo hasta que la comunidad científica establezca, desde la perspectiva que da la distancia con los acontecimientos, una serie de consensos en cada campo de estudio sobre lo acontecido en estos meses.

A día de hoy, se tiene constancia de que es a finales de 2019, en la ciudad China de Wuhan, cuándo y dónde se comienzan a detectar un número considerable de neumonías provocadas por un virus del tipo coronavirus, el SARS-CoV2. La enfermedad, denominada por la OMS como COVID-19, es la primera epidemia mundial del mundo moderno (Gou et al., 2020, p.2; Ramos, 2020, p. 225). Hasta la fecha, el origen de la infección se localiza en el mercado de mariscos de Huanan. Si bien los científicos aún no han podido identificar con total fiabilidad la fuente animal de la que mutó el virus siendo transferida al ser humano, son varios los autores que sospechan del murciélago (Palacios et al., 2020: p.2; Shereen et al., 2020: p.96). Debido a su alta contagiosidad y rápida propagación, el virus se extendió de forma inmediata a más de 100 países (Shereen et al., 2020, p.96). Tras una respuesta inicial tímida, y conforme el virus se convertía en una amenaza real para el colapso de sus sistemas sanitarios, casi todos los gobiernos comenzaron a tomar decisiones drásticas para evitar el contagio masivo. Según los primeros estudios de los que tenemos constancia, se observa que el 31 por ciento de los pacientes ingresados con coronavirus tienen problemas para respirar, y que en un 75 por ciento de los casos aparecen neumonías bilaterales. Dos de 
las manifestaciones clínicas más comunes que se observan en los pacientes son la aparición de fiebre y tos, dándose ambas en más de un 80 por ciento de los afectados (Rothan y Byrareddy, 2020, p. 1 ; Palacios et al., 2020, p.4).

En España se decretó el estado de alarma el 14 de marzo de 2020 (Gómez, 2020, p. 29), y los menores no pudieron salir de sus domicilios al menos hasta el domingo 26 de abril de 2020, día en el que se levantó para ellos, y solo parcialmente, el confinamiento. Entre estas dos fechas hemos recogido los datos referentes a este estudio a través de un cuestionario que se ha sometido vía online dirigido a sus padres. Además, el 13 de marzo fue el último día en que los niños y niñas asistieron a la escuela y tuvieron contacto social directo con otras personas que no pertenecieran a su núcleo familiar más próximo, con quienes conviven. En total han sido 43 días sin salir de sus domicilios, restricción que apenas han esquivado aquellos menores con patologías muy específicas, a quienes se ha permitido pasear acompañados por un adulto. Solo a partir de entonces ha comenzado una desescalada progresiva, pudiendo salir los menores 1 hora al día a pasear junto a un adulto, manteniendo, eso sí, las medidas de higiene, protección y distancia social adecuadas. Este hecho, creemos, cambia cualitativamente la situación de los menores con respecto a las semanas anteriores, razón por la cual decidimos acabar aquí la recolección de los datos que conforman nuestra muestra.

Este estudio carece de antecedentes concretos, dado que el confinamiento social del país es una experiencia sin precedentes históricos en el mundo moderno. Aunque se han producido pandemias víricas en otras ocasiones, hasta el día de hoy nunca habían implicado a todos los continentes al mismo tiempo, ni habían resultado ser víricamente tan contagiosas. La excepcionalidad del fenómeno hace que resulte muy complicado encontrar investigaciones que analicen los problemas que puedan surgir en tales circunstancias. Por las semejanzas que tiene con respecto a la interrupción de la vida normal de los individuos, recurrimos a investigaciones relacionadas con el aislamiento que se produce cuando un menor es hospitalizado para guiar nuestro estudio sobre qué efectos podrían derivarse del aislamiento social fruto del confinamiento.

La hospitalización se caracteriza por la interrupción de la vida cotidiana. Paraliza casi todas las relaciones del individuo con el entono, ya sean a nivel familiar, escolar y social (Lizasoáin y Ochoa, 2003, p.76; Hernández y Rabadán, 2013 , p. 168). Si bien existen factores lógicamente asociados con el ingreso en el hospital, esta suspensión de su vida normal conlleva la aparición de aspectos psicológicamente adversos que alteran la cognición, las emociones y las conductas de los individuos (Cruz, Mejías y Machado, 2014, p. 464). Algunas de estas alteraciones pueden ser: comportamientos agresivos; trastornos del sueño; dependencia afectiva; comportamientos evitativos; trastornos del apetito; dificultades para concentrarse; ansiedad; miedo; temor; tristeza; depresión; y apatía (Lizasoáin y Ochoa, 2003, pp.79-80; Lizasoáin y Polaino-Lorente, 1992, p.57; Gutiérrez, 2017, p. 166). Otros autores afirman que en los niños de más temprana edad pueden aparecer: llantos; inquietud; y conductas regresivas, entre otros síntomas (del Barrio y Mestre, 1989, p.86).

De entre todas las alteraciones citadas la ansiedad ocupa un lugar destacado en la literatura, apareciendo con bastante frecuencia en la hospitalización pediátrica como causa de una serie de factores limitantes que perjudican el aprendizaje de los menores (López-Naranjo y Fernández-Castillo, 2006, p.571). En el caso que es objeto de análisis, sí es cierto que los menores continúan manteniendo un nexo con el ambiente educativo propio a través de la figura del profesor o profesora y no una desconexión total; por ejemplo, los menores reciben clases online o reciben material para realizar tareas en sus casas. Sin embargo, la sensación de ansiedad derivada de este contacto meramente virtual, al que no están habituados en un momento como este, junto con el aislamiento 
social y la ausencia de relación directa con los compañeros y compañeras de clase, puede tener unos efectos similares para el aprendizaje.

Debemos tener en cuenta que las diferencias ambientales en ambos casos pueden ser importantes para los resultados obtenidos. Los menores hospitalizados pasan a vivir en un ambiente "hostil", desconocido para ellos como son las habitaciones del hospital, lo que promueve una serie de hándicaps que afectan al impacto psicológico del menor (Lizasoáin y Ochoa, 2003, p.78). En este sentido, el confinamiento de los menores no tiene lugar en ambientes desconocidos, sino en sus propios hogares. Están en un lugar seguro y conocido, circunstancia que es cualitativamente muy diferente. Otro factor que claramente puede motivar resultados distintos es el temor a procesos dolorosos como los asociados al ingreso hospitalario derivados de la enfermedad. Esto incluye el dolor o malestar propio de la enfermedad, pero también el dolor asociado al tratamiento médico, derivado este de las pruebas e intervenciones médicas que son percibidas como amenazantes, dolorosas y peligrosas por ellos (Fernández-Castillo y López-Naranjo, 2006, p.633; López-Naranjo y Fernández-Castillo, 2004, p.4).

\section{ARTE Y CREATIVIDAD: ENTRE LA AUTONOMÍA Y EL HECHO SOCIAL}

El arte es un elemento que lleva entre nosotros desde épocas inmemorables. Debido a su alta capacidad multidisciplinar, se considera un término complejo que ha ocasionado discusiones apasionadas entre los distintos profesionales desde la perspectiva correspondiente de cada disciplina (Granados, 2009, p.52; Eco, 1970, 127). El estudio del papel social jugado por el arte aparece con las primeras huellas del ser humano. Klein $(2012$, p.30) identifica la unión entre arte y objetivos terapéuticos desde la prehistoria. Este hecho sugiere que el arte ha estado unido y ligado al desarrollo del individuo desde los inicios de la humanidad.

Del mismo modo, la creatividad aparece en edades muy tempranas. Tanto es así que Erro- teta $(2011$, p.29) sitúa el primer acto creador del individuo en el "chupeteo"; es decir, en el acto que se produce después de haberse alimentado a través del pecho de la madre y ser apartado de él. La respuesta del bebé al no obtener aquello que demanda es la sustitución del objeto faltante. Ello le sirve para calmar el llanto y autosatisfacerse, desarrollando así el primer acto de creación, el cual aparece en forma de alucinación que le permite sustituir el objeto deseado. Como ilustra este ejemplo, la creatividad acompaña al individuo desde la primera infancia. En edades más avanzadas, la facultad de crear se desarrolla a través de los juegos que realizan los menores (Vigotsky, 2003, p.5), para quienes el juego es una herramienta de descubrimiento, aprendizaje y autoconocimiento muy importante en el día a día; y que produce en los individuos satisfacción (Fernández, 2003, p. 140). Estos actos lúdicos son imprescindibles para su desarrollo social, comunicativo y espiritual (de Valdenebro, 2001, p.63). En este artículo no vamos a profundizar en el juego, o en la relación entre el arte y el juego. Bastará apuntar que la realización de actividades artísticas es un acto de experimentación autónomo que contiene en sí mismo un proceso de descubrimiento individual y un elemento de juego, que puede cubrir los aspectos involucrados en momentos donde no podemos acceder a él.

El proceso creador y su funcionamiento, tanto desde el punto de vista patológico como saludable, ha suscitado un gran interés. Hoy día se considera que una persona creativa tiene más capacidad para adaptarse cognitiva y afectivamente al medio y a los cambios (Granados, 2009 , p.54). Es por esta razón que haremos referencia al acto creador tal y como es planteado por autores como Fernández-Cao (2011), quien afirma que un individuo que se enfrenta a algo nuevo e imprevisto tiende a cuestionarlo y rechazarlo. La posibilidad de adaptarse a esa novedad es brindada por la creatividad; esto es, la facultad de crear es desarrollada como una herramienta que amplía las capacidades y habilidades del individuo, que fomenta su flexibilidad permitiéndole una búsqueda 
de soluciones que le permiten adaptarse a lo nuevo (Fernández-Cao, 201 1, p.20 y 25).

Vigotsky (2003, p.4) considera que una persona creativa tiene la vista puesta hacia el futuro y es capaz de intervenir en su presente. El arte muestra al individuo su propia realidad, le permite comunicarse y expresarse reflejando sus emociones sin necesidad de verbalizar. Incluso las creaciones imaginarias son "modificaciones de lo empíricamente presente", como advierte Adorno (1970 [1983], p.34). Pintar, por ejemplo, es un acto que permite al individuo entender su mundo y lo que le rodea; le permite ser; le permite aunar lo particular y lo colectivo, y es de este modo que el individuo parte de sí para relacionarse con el otro (Fernández-Cao, 2011 1, pp. 19, 23 y 33). Desde el garabato de los más pequeños a los dibujos o pinturas más elaboradas de los adolescentes, independientemente de las habilidades artísticas que posean; e incluso carentes de la búsqueda consciente de una belleza estética; cada creación tiene una intención y un motivo subyacente. Como lo expresa Fernández-Cao (201 1, p.33) o Lotman (1982), las creaciones artísticas son actos de comunicación. Winnicott (1971, p.115) va mucho más allá y señala que es la creatividad lo que hace que el individuo perciba que la vida merece la pena.

\section{TRABAJO EMPÍRICO: ARTE, EMOCIONES Y COVID-19}

\section{METODOLOGÍA Y}

\section{CARACTERÍSTICAS DE LA MUESTRA}

El método utilizado para esta investigación es la encuesta. Se ha diseñado un cuestionario siguiendo la evidencia mostrada por la literatura relacionada con la hospitalización pediátrica, pues creemos que muchas observaciones pueden adaptarse, en virtud de su semejanza más que de sus diferencias, a la situación de confinamiento motivada por la epidemia. El cuestionario se lanzó el día 8 de abril de forma online, referente al estudio emocional de los menores y a las implicaciones que la realización de actividades artísticas está teniendo sobre su comportamiento y bienestar emocional durante el confinamiento en su etapa íntegra. El período de recogida de datos se extendió, concretamente, desde el 8 de abril hasta el 26 de abril, día, este último, en que se optó por no recoger más datos, dado el levantamiento parcial del confinamiento para los menores (a partir de entonces, los menores podían ya salir a pasear durante 1 hora diaria). No tener en cuenta este cambio y seguir con la recolección de datos habría distorsionado, según nuestro criterio, los resultados del estudio.

Respondieron al cuestionario un total de 48 padres y madres. El conjunto de sujetos que forman la muestra son 75. La diferencia entre el número de la muestra y el de los cuestionarios se produce porque en algunas viviendas se encuentran confinados más de un menor. Con estas 75 observaciones se crea la base de datos codificando las respuestas declaradas en los cuestionarios online.

El cuestionario contiene unas primeras preguntas de clasificación para presentar la composición de la muestra por edades, sexo, si habían salido a la calle o no durante los días en que el encierro absoluto se mantuvo, y si tenían alguna patología. Reflejamos brevemente las características de la muestra en función de estas características. Primero, debe indicarse que existe una amplia variedad de edades. En las edades comprendidas entre 1 y 5 años, correspondiente a la etapa de educación infantil, nos encontramos con 30 observaciones, 11 de ellas relativas a menores con 5 años. En las edades incluidas entre 6 y 12 años, referente a la etapa de educación primaria, disponemos de 36 observaciones. En relación a la etapa adolescente, que abarca desde los 13 a los 17 años, solo tenemos 9 observaciones. Segundo, en lo relativo al sexo, del conjunto de la muestra prácticamente la mitad son niños (49 por ciento) y la otra mitad son niñas (51 por ciento). Tercero, la gran mayoría de los menores no han salido en ningún momento a la calle durante el tiempo de confinamiento (en concreto, un 92 por ciento, frente a un 8 por ciento que sí ha salido, aunque sea puntualmente). Además, de este bajo porcentaje 
menos de la mitad de los que han indicado haber salido declara que el niño o niña tenía alguna patología, que son el 3 por ciento de la muestra. Si bien ninguno de ellos declaraba tener una de las patologías indicadas como excepción bajo el estado de alarma para salir de su domicilio.

\section{SÍNTOMAS APRECIADOS DURANTE LA CUARENTENA}

Dado que el confinamiento decretado es un suceso excepcional y supone una interrupción de la vida cotidiana, tanto para los adultos como para los menores, a través de esta investigación hemos identificado los posibles síntomas físicos y emocionales que la cuarentena puede causar y su incidencia en este segundo grupo. Para ello se ha creado el Gráfico 1 con la información obtenida de los cuestionarios. Observamos que casi un tercio de los niños y niñas de la muestra no han padecido ningún síntoma; mientras que los dos tercios restantes sí, en buena parte de los casos más de uno. De mayor a menor, los síntomas observados por los padres en los menores son: alteraciones del sueño $(42,67$ por ciento); dolor de cabeza (28 por ciento); cambios en los hábitos alimenticios (25,33 por ciento); disminución del apetito $(10,67$ por ciento); pesadillas (8 por ciento); ha vuelto a mojar la cama $(6,67$ por ciento); y dolor de estómago (6,67 por ciento).

Dolor de cabeza.

Disminución del apetito.

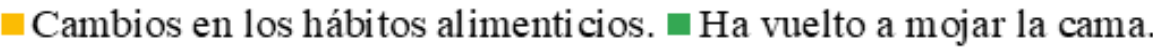

Tiene pesadillas.

- Alteración del sueño.

- Dolor de estómago.

- Ninguna.
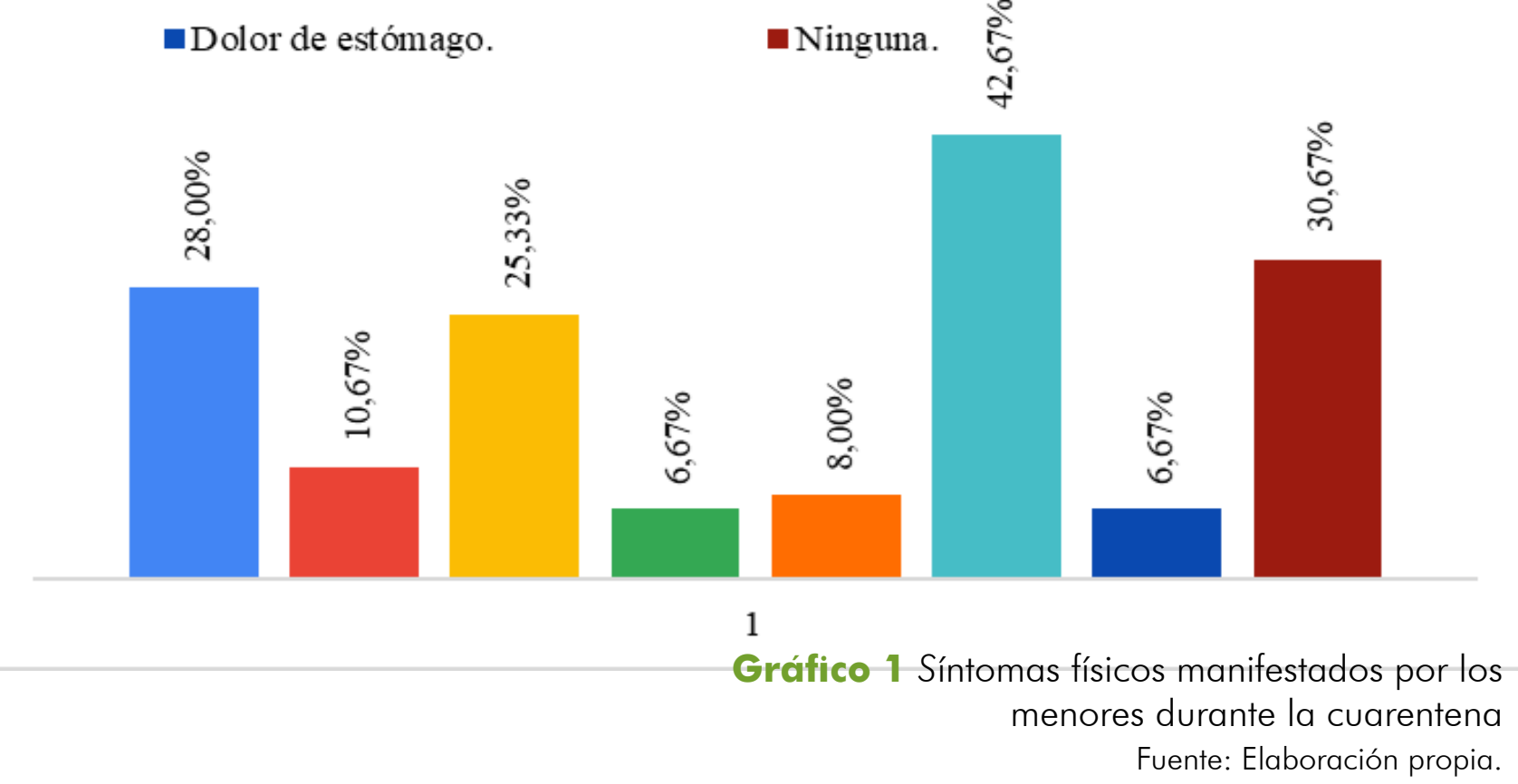
Otro aspecto que hemos identificado se refiere a aquellas emociones o comportamientos que han mostrado los menores encerrados en sus hogares durante este largo período. En el Gráfico 2 se presentan los síntomas emocionales que han manifestado los menores durante la cuarentena. El 17,33 por ciento de los padres afirman no haber observado ningún tipo de síntoma emocional en ellos; mientras que una amplia mayoría, el 82 por ciento, ha respondido de modo afirmativo marcando varios de estos síntomas. De mayor a menor, los síntomas emocionales observados declarados son: nerviosismo (41,3 por ciento); apego excesivo con el adulto (37,33 por ciento); negación a participar en actividades familiares y tristeza $(17,33$ por ciento); llantos constantes (16 por ciento); incapacidad para relajarse (14,67 por ciento); rabia (14,67 por ciento): agresividad $(13,33$ por ciento); regresión a comportamientos de etapas anteriores e incapacidad de controlar sus emociones (13,33 por ciento); preocupaciones y miedos recurrentes (12 por ciento); ansiedad (9,33 por ciento); ha reducido su dedicación al juego (8 por ciento); tristeza $(6,67$ por ciento); incapacidad para controlar sus emociones (5,33 por ciento); y comportamientos auto lesivos (1,33 por ciento).

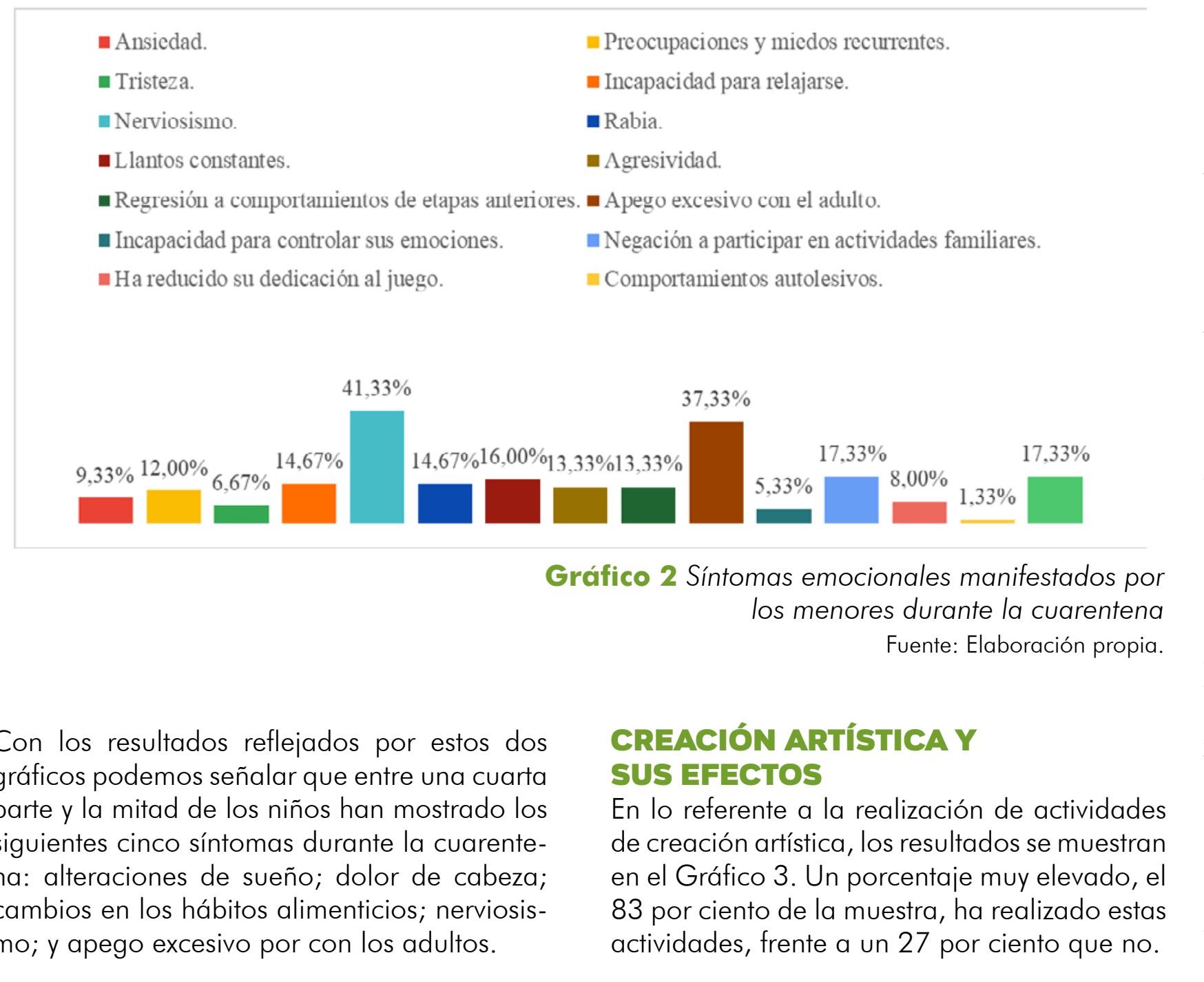




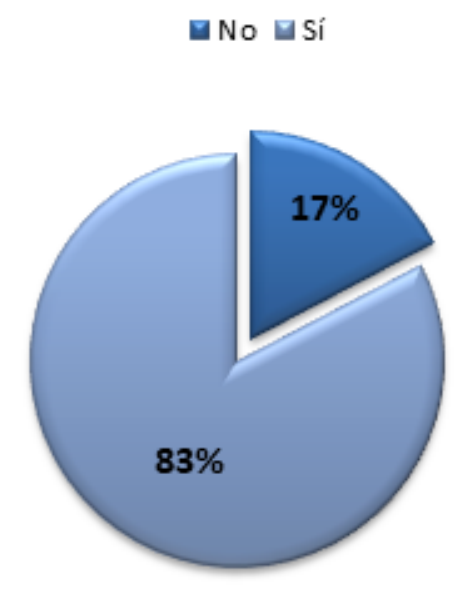

Gráfico 3. Realización de actividades artísticas durante la cuarentena Fuente: Elaboración propia.

Si comparamos estas cifras con las mostradas en el Gráfico 4 se deriva un dato interesante: un porcentaje mucho menor, el 48 por ciento, asistía a iniciativas creativas de forma regular antes de que el estado de alarma se declarase; esto es, un 35 por ciento más de niños y niñas realizan actividades artísticas durante el confinamiento que antes del mismo no realizaban.

\section{No $\square$ Sí}

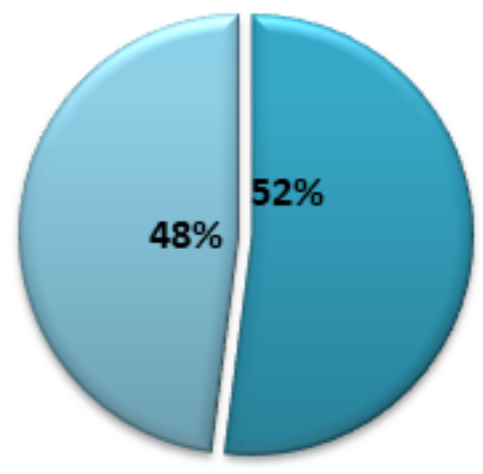

Gráfico 4. Realización de actividades artísticas antes de la epidemia Fuente: Elaboración propia.
Dada la gran diferencia entre los sujetos que realizan actividades artísticas en sus domicilios que observamos en el Gráfico 3 y la asistencia o participación en actividades artísticas antes del confinamiento que ilustra el Gráfico 4, vemos interesante observar el tiempo semanal que estos menores dedican a ello durante su encierro. Esto lo hacemos en el Gráfico 5.

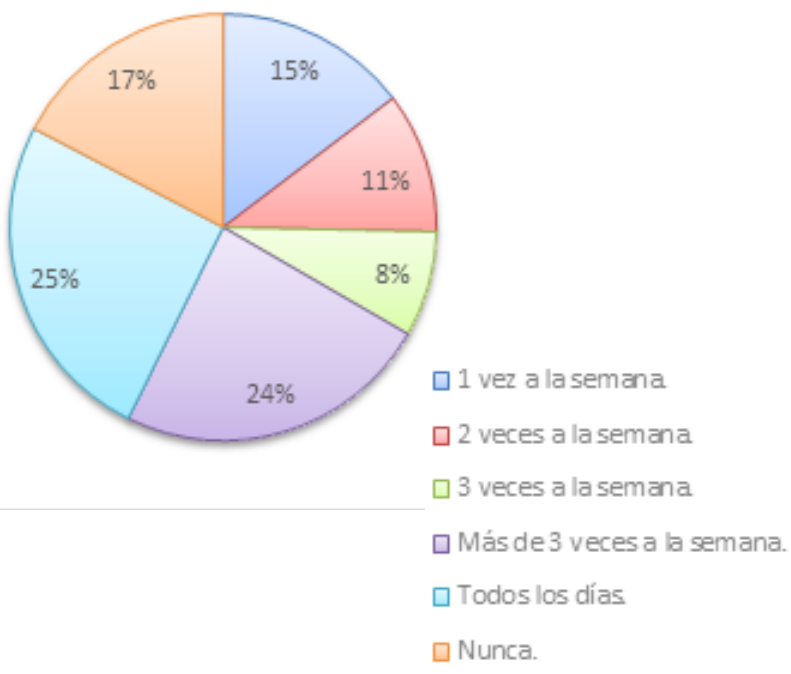

Gráfico 5. Tiempo dedicado a la realización de actividades artísticas durante la cuarentena Fuente: Elaboración propia.

En el Gráfico 5 observamos que un 8 por ciento de los menores hace alguna creación al menos 3 veces a la semana, un 24 por ciento de la muestra más de 3 veces por semana, y un 25 por ciento lleva a cabo dichas actividades de forma diaria. En total un 57 por ciento desarrolla este tipo de actividades de forma más o menos cotidiana, lo que se acerca al 48 por ciento que declaran haber asistido a actividades artísticas o talleres habitualmente antes de la declaración del estado de alarma que conllevó al confinamiento.

Por último, recogemos en el Gráfico 6 los beneficios que han sido detectados producidos por las actividades de creación artística que los menores han desarrollado en sus domicilios. 


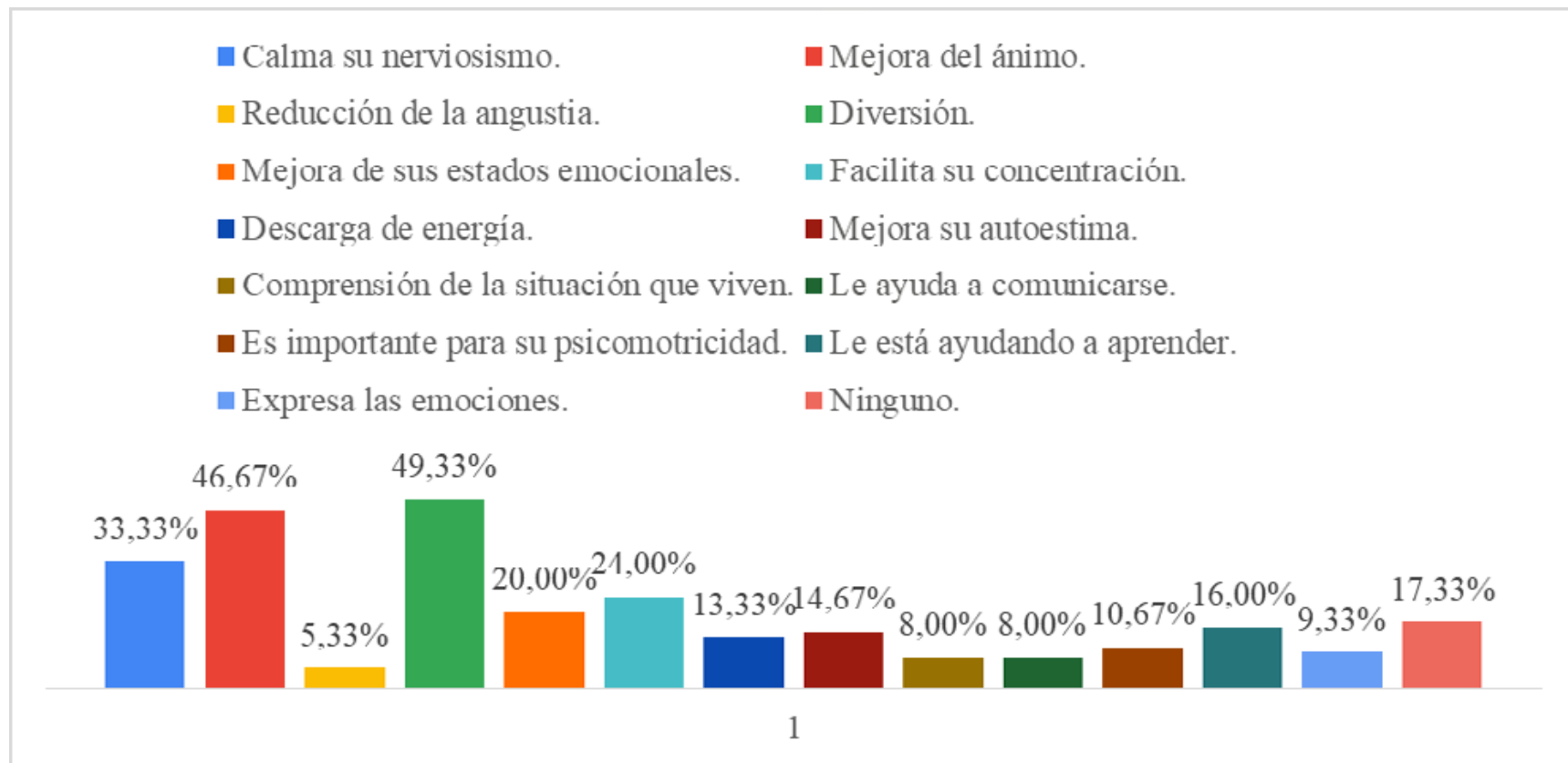

Gráfico 6 Beneficios declarados tras la realización de actividades artísticas

Nota: Se amplían los resultados con los Anexos 1 y 2 , referentes a la correlación de los beneficios declarados tras la realización de actividades artísticas con, primero, los síntomas físicos mostrados por los menores durante la cuarentena reflejados en el Gráfico 1; y segundo, con los síntomas emocionales mostrados por los menores que son ilustrados en el Gráfico 2. Todo ello es reflejado en las Tablas 2 y 3 , respectivamente.

Fuente: Elaboración propia.

En primer lugar, hay que mencionar que el 17,33 por ciento de quienes declaran no haber observado ningún tipo de beneficio en los menores coincide con el porcentaje que en las preguntas previas señalaron no haber realizado ninguna actividad. Esto nos sugiere que los datos recolectados, aunque menos cuantiosos de los que nos hubiera gustado, han sido suministrados correctamente por los padres, sin errores. El 82,67 por ciento restante, la gran mayoría de los encuestados, manifiesta haber observado en sus hijos los siguientes benefi- cios: diversión $(49,33$ por ciento); mejora del ánimo $(46,67$ por ciento); calma sus nerviosismo (33,33 por ciento); mejora de su concentración (24 por ciento); mejora de sus estados emocionales (20 por ciento); ninguno (17,33 por ciento) le está ayudando a aprender (16 por ciento); mejora de su autoestima $(14,67$ por ciento); descarga de energía (13,33 por ciento); es importante para su psicomotricidad (10,67 por ciento); expresa las emociones (9,33 por ciento); comprensión de la situación que viven ( 8 por ciento); le ayuda a comunicarse (8 por ciento); y en un menor porcentaje, reducción de la angustia (5,33 por ciento).

Para mostrar la relación entre los beneficios declarados tras la realización de las actividades artísticas con los síntomas físicos y emocionales manifestados hemos estimado las correlaciones entre las distintas variables, las cuales pueden observarse en las Tablas 1 y 2. 
Tabla 1. Correlaciones entre síntomas físicos manifestados y beneficios declarados tras la realización de actividades artísticas

\begin{tabular}{lccccccc} 
& CABEZ & PESAD & ALIME & ESTOM & APETI & MOJAR & SUEÑO \\
\hline ANGUS & 0,12 & $-0,07$ & 0,13 & $-0,06$ & 0,30 & $-0,06$ & $-0,20$ \\
\hline ÁNIMO & 0,07 & 0,32 & $-0,11$ & 0,18 & $-0,32$ & $-0,04$ & $-0,10$ \\
\hline APREN & $-0,03$ & 0,14 & $-0,09$ & 0,17 & $-0,15$ & 0,32 & 0,14 \\
\hline ESTIM & $-0,01$ & 0,16 & $-0,07$ & 0,34 & $-0,14$ & $-0,11$ & $-0,05$ \\
\hline CALMA & 0,44 & 0,21 & $-0,22$ & 0,26 & 0,49 & $-0,19$ & 0,02 \\
\hline COMPR & 0,04 & $-0,09$ & $-0,17$ & $-0,08$ & $-0,10$ & $-0,08$ & $-0,25$ \\
\hline COMUN & 0,25 & 0,28 & $-0,17$ & $-0,08$ & $-0,10$ & $-0,08$ & $-0,25$ \\
\hline CONCE & 0,34 & 0,29 & $-0,11$ & 0,10 & 0,41 & $-0,03$ & $-0,04$ \\
\hline DIVER & 0,22 & 0,10 & $-0,15$ & 0,27 & 0,18 & 0,27 & 0,17 \\
\hline ENERG & 0,28 & 0,46 & $-0,23$ & 0,52 & 0,12 & $-0,10$ & $-0,02$ \\
\hline EXPRE & 0,00 & 0,24 & 0,34 & $-0,09$ & $-0,11$ & $-0,09$ & $-0,28$ \\
\hline MEJOR & 0,28 & 0,34 & $-0,29$ & 0,13 & $-0,17$ & 0,27 & 0,04 \\
\hline PSICO & $-0,02$ & 0,22 & $-0,10$ & $-0,09$ & 0,16 & 0,43 & 0,23
\end{tabular}

Nota: Dolor de cabeza (CABEZ); tiene pesadillas (PESAD); cambios en los hábitos alimenticios (ALIME); dolor de estómago (ESTOM); disminución del apetito (APETI); ha vuelto a mojar la cama (MOJAR); alteración del sueño (SUEÑO); reducción de la angustia (ANGUS); mejora del ánimo (ÁNIMO); le está ayudando a aprender (APREN); mejora su autoestima (ESTIM); calma su nerviosismo (CALMA); comprensión de la situación que viven (COMPR); le ayuda a comunicarse (COMUN); facilita su concentración (CONCE); diversión (DIVER); descarga de energía (ENERG); expresa sus emociones (EXPRE); mejora sus estados emocionales (MEJOR); y es importante para su psicomotricidad (PSICO).

Fuente: Elaboración propia.

Con respecto a los síntomas físicos declarados, la Tabla 1 refleja algunas relaciones entre éstos y los beneficios de pintar que, estadísticamente, exponemos que pueden darse juntos en función de los datos que hemos recogido en los cuestionarios. Así, por ejemplo, realizar actividades creativas está relacionado de forma positiva con calmar el nerviosismo y facilitar la concentración para aquellos que han declarado tener como síntoma el tener recurrentes dolores de cabeza; o descargar energía y mejorar el estado de ánimo también se correlaciona de forma positiva con aquellos que declaran que sus hijos tienen pesadillas. 
Tabla 2Correlaciones entre síntomas emocionales manifestados y beneficios declarados tras la realización de actividades artísticas

\begin{tabular}{llllllll} 
& ANSIE & MIEDO & TRIST & RELAJ & NERVI & RABIA & REGRE \\
\hline ANGUS & $-0,08$ & $-0,09$ & $-0,07$ & $-0,10$ & 0,28 & 0,24 & $-0,10$ \\
\hline ÁNIMO & 0,25 & 0,39 & 0,28 & $-0,10$ & $-0,05$ & 0,06 & 0,02 \\
\hline APREN & 0,23 & $-0,17$ & 0,17 & $-0,19$ & $-0,08$ & 0,02 & 0,25 \\
\hline ESTIM & $-0,14$ & 0,42 & 0,04 & 0,04 & $-0,05$ & 0,04 & 0,17 \\
\hline CALMA & $-0,04$ & $-0,01$ & 0,03 & 0,02 & 0,37 & 0,34 & $-0,12$ \\
\hline COMPR & $-0,10$ & $-0,11$ & $-0,08$ & $-0,13$ & $-0,06$ & $-0,13$ & $-0,12$ \\
\hline COMUN & $-0,10$ & $-0,11$ & $-0,08$ & 0,15 & 0,15 & $-0,13$ & $-0,12$ \\
\hline CONCE & $-0,19$ & $-0,02$ & $-0,16$ & $-0,06$ & 0,41 & 0,11 & 0,05 \\
\hline DIVER & $-0,03$ & $-0,19$ & $-0,04$ & $-0,10$ & $-0,05$ & 0,06 & 0,34 \\
\hline ENERG & 0,14 & 0,09 & 0,21 & 0,28 & 0,14 & 0,28 & 0,07 \\
\hline EXPRE & $-0,11$ & $-0,12$ & $-0,09$ & 0,12 & 0,19 & $-0,14$ & $-0,13$ \\
\hline MEJOR & 0,06 & $-0,19$ & 0,13 & $-0,02$ & $-0,03$ & $-0,02$ & 0,29 \\
\hline PSICO & $-0,11$ & $-0,13$ & $-0,10$ & 0,10 & $-0,04$ & $-0,15$ & 0,24 \\
\hline & LLANT & AGRES & APEGO & $-10 E G 0$ & ACTFA & LESIO & NOCTR \\
\hline ANGUS & $-0,11$ & 0,60 & $-0,19$ & $-0,07$ & 0,20 & $-0,03$ & $-0,06$ \\
\hline ÁNIMO & 0,31 & $-0,06$ & 0,15 & $-0,29$ & $-0,16$ & $-0,11$ & 0,01 \\
\hline APREN & 0,20 & 0,04 & 0,03 & $-0,13$ & 0,08 & $-0,05$ & $-0,11$ \\
\hline ESTIM & 0,23 & $-0,06$ & 0,22 & $-0,13$ & $-0,20$ & $-0,05$ & $-0,10$ \\
\hline CALMA & $-0,01$ & 0,38 & $-0,39$ & 0,10 & $-0,03$ & $-0,09$ & $-0,17$ \\
\hline COMPR & $-0,13$ & 0,17 & $-0,03$ & $-0,09$ & $-0,14$ & $-0,04$ & $-0,07$ \\
\hline COMUN & $-0,13$ & 0,17 & $-0,24$ & $-0,09$ & 0,12 & $-0,04$ & $-0,07$ \\
\hline CONCE & $-0,08$ & 0,14 & $-0,19$ & $-0,06$ & 0,15 & $-0,07$ & $-0,14$ \\
\hline DIVER & 0,31 & 0,18 & 0,20 & $-0,09$ & $-0,23$ & $-0,11$ & $-0,23$ \\
\hline ENERG & 0,25 & 0,30 & $-0,15$ & 0,17 & 0,02 & $-0,05$ & $-0,10$ \\
\hline EXPRE & $-0,14$ & $-0,13$ & $-0,07$ & $-0,10$ & 0,33 & $-0,04$ & $-0,08$ \\
\hline MEJOR & 0,14 & 0,19 & $-0,05$ & $-0,15$ & $-0,06$ & $-0,06$ & $-0,12$ \\
\hline PSICO & 0,08 & 0,37 & 0,08 & 0,37 & $-0,16$ & $-0,04$ & $-0,08$ \\
\hline
\end{tabular}

Nota: Ansiedad (ANSIE); preocupaciones y miedos recurrentes (MIEDO); tristeza (TRIST); incapacidad para relajarse (RELAJ); nerviosismo (NERVI); rabia (RABIA); regresión a comportamientos de etapas anteriores (REGRE); llantos constantes (LLANT); agresividad (AGRES); apego excesivo con el adulto (APEGO); ha reducido su dedicación al juego (JUEGO); negación a participar en actividades familiares (ACTFA); comportamientos autolesivos (LESIO); ninguna (NOCTR); reducción de la angustia (ANGUS); mejora del ánimo (ÁNIMO); le está ayudando a aprender (APREN); mejora su autoestima (ESTIM); calma su nerviosismo (CALMA); comprensión de la situación que viven (COMPR); le ayuda a comunicarse (COMUN); facilita su concentración (CONCE); diversión (DIVER); descarga de energía (ENERG); expresa sus emociones (EX$\mathrm{PRE})$; mejora sus estados emocionales (MEJOR); y es importante para su psicomotricidad (PSICO).

Fuente: Elaboración propia 
La Tabla 2 ilustra los beneficios que están estadísticamente relacionados con los síntomas emocionales declarados. Analizando las relaciones más destacadas, vemos que tras realizar actividades creativas existe una relación positiva en la mejora del ánimo y la autoestima cuando los menores manifiestan tener preocupaciones y miedos recurrentes. También que existen beneficios positivos en la concentración y la calma del nerviosismo de aquellos que expresan nerviosismo. Igualmente, con relación a los menores que manifiestan una regresión a comportamientos de etapas anteriores y un llanto constante, realizar este tipo de actividades parece que le aporta diversión, aprendizaje y mejora su estado de ánimo. Aquellos que manifiestan agresividad, la relación de beneficios observada apunta fundamentalmente a reducir su angustia. Por último, también parece verificarse la relación inversa entre quienes declaran que la creatividad facilita su concentración y manifiestan un apego excesivo por el adulto.

\section{CONCLUSIONES}

Los 43 días de confinamiento obligatorio íntegro que van desde el 14 de marzo al 26 de abril de 2020 han supuesto la interrupción de los procesos de socialización, aprendizaje y desarrollo cognitivo habituales de los menores. Estos se han visto obligados a permanecer en casa por motivos sanitarios, y han visto suspendidas sus clases, sus actividades recreativas y el contacto con sus compañeros, e incluso se les ha privado de estar cerca de otros ciudadanos o familiares. Todas estas circunstancias hacen que los menores hayan visto alteradas y modificadas sus rutinas, sus vidas, y hayan tenido que buscar recursos adaptativos para poder asumir y afrontar estos cambios. En los resultados presentados detectamos los síntomas producidos por el confinamiento. Dada la inexistencia de literatura con los que compararlos en el contexto de un fenómeno de la misma naturaleza, hemos pensado que estos pueden ser confrontados con los síntomas observados durante la hospitalización pediátrica, pues esta deviene en una situación de aislamiento social que también interrumpe los pro- cesos normales de los menores. Este ejercicio lo hemos sintetizado en la Tabla 3, que merece una reflexión.

Tabla 3Comparación de los síntomas en menores fruto del aislamiento social

\begin{tabular}{lll}
\multicolumn{1}{c}{ Síntomas } & Cuarentena & $\begin{array}{c}\text { Hospitalización } \\
\text { pediátrica }\end{array}$ \\
\hline $\begin{array}{l}\text { Comportamientos } \\
\text { agresivos }\end{array}$ & $X$ & $X$ \\
\hline Trastornos del sueño & $X$ & $X$ \\
\hline Trastornos del apetito & $X$ & $X$ \\
\hline Dependencia afectiva & $X$ & $X$ \\
\hline Comportamientos & $X$ & $X$ \\
evitativos & $X$ & $X$ \\
\hline Falta de concentra- & $X$ & $X$ \\
ción & $X$ & $X$ \\
\hline Ansiedad & $X$ & $X$ \\
\hline Miedo & -- & $X$ \\
\hline Temor & $X$ & $X$ \\
\hline Tristeza & $X$ & $X$ \\
\hline Depresión & -- & $X$ \\
\hline Apatía & $X$ & $X$ \\
\hline Llantos & $X$ & -- \\
\hline Inquietud/nerviosismo & $X$ & $X$ \\
\hline Conductas regresivas & $X$ & $X$ \\
\hline Dolor de cabeza & $X$ & $X$ \\
\hline Pesadillas & $X$ & $X$ \\
\hline Dolor de estomago & $X$ & $X$ \\
\hline
\end{tabular}

Fuente: Elaboración propia.

Como puede deducirse de dicha tabla, los síntomas causados por el confinamiento guardan una similitud significativa con los síntomas que produce la hospitalización pediátrica. Creemos que estas semejanzas se deben a que en ambos casos se comparten aspectos como el aislamiento social, la imposición de permanecer en un espacio cerrado, y la interrupción de la vida cotidiana. Es cierto que el menor hospitalizado tiene otras circunstancias que agudizan los síntomas identificados; pero dichas circunstancias se corresponden con la propia condición de enfermo, procediendo del hecho de vivir en un contexto hospitalario o de las múltiples pruebas a las que están sometidos. 
Estos factores no son enfrentados por los menores bajo la cuarentena declarada a causa de la epidemia del COVID-19, ya que su confinamiento se produce en un entorno seguro y conocido como es el hogar.

Teniendo en cuenta que el arte ayuda al ser humano a la búsqueda de soluciones, le permite expresarse y entender el mundo que le rodea (Fernández-Cao, 2011 , p.33), podemos inferir que ésta puede ser una buena razón para explicar la elevada actividad creativa observada en los hogares durante la cuarentena, donde un 83 por ciento de los hogares encuestados afirma que los menores han realizado en algún momento alguna actividad artística. Estas actividades no son un suceso puntual ya que el 57 por ciento de la muestra manifiesta realizar creaciones artísticas un mínimo de 3 veces semanales. La regularidad declarada puede deberse a la necesidad de comunicación de los menores en una situación anómala que ha trastornado sus vidas profundamente. En un momento donde la consigna es el aislamiento social, dibujar o pintar lo que nos sucede sin la necesidad de poner palabras, en edades más tempranas, es "más fácil", ya que la identificación de emociones es algo complejo para el que no todos los menores cuentan con el vocabulario o capacidad oral de expresión oportunos.

Por último, nos resulta relevante para verificar la hipótesis de nuestro estudio que el 83 por ciento de los menores realicen actividades artísticas en sus domicilios cuando únicamente el 52 por ciento de la muestra expone el haber asistido a clases o talleres artísticos antes del confinamiento y de forma cotidiana. No obstante, debemos remarcar que esa diferencia puede deberse a tres elementos: 1) a la prevalencia en la muestra de menores en edades más tempranas, que por su corta edad aun no utilizan ciertos materiales debido a su carencia de habilidades y a estar aún en proceso de aprendizaje; 2) al hecho de que estas actividades suelen tener un carácter privado, por lo que entra en juego el poder adquisitivo de las familias; y 3) a la mayor necesidad creadora que deriva de las circunstancias excepcionales del encierro.

Además de la evidencia aquí presentada sobre los numerosos beneficios del proceso de creación artística, existe evidencia de que entre las familias de rentas bajas la propensión a la aparición de trastornos de salud mental es superior (Gili et al., 2013; Urbanos-Garrido y López-Valcárcel, 2015); que las audiencias de las artes están sesgadas hacia la derecha en cuanto a ingresos y niveles de educación (Baumol y Bowen, 1966; Ateca-Amestoy y Prieto-Rodríguez, 2013; Falk y Katz-Gerro, 2016); así como también que la educación artística temprana es la variable fundamental que explica la participación posterior en y el consumo de productos y servicios culturales, independientemente de las diferencias en los ingresos (O'Hagan, 1996; Blaug, 2001; Pérez-Villadoniga y Suárez-Fernández, 2019). Como apuntan Pérez-Villadoniga y Suárez Fernández (2019, p.102), la educación temprana para desarrollar los intereses y gustos artísticos es el factor principal para impulsar la participación en actividades culturales, en lugar de la reducción de impuestos. Esto es así porque, normalmente, estas actividades implican la interpretación de contenidos simbólicos para los cuales uno debe estar entrenado (Suárez-Fernández, Prieto-Rodríguez, y Pérez-Villadoniga, 2020). Por ello, la reducción de impuestos sobre el valor añadido en estos productos y servicios es altamente regresiva; mientras que la búsqueda de diversas formas de acceso a una educación artística temprana para todos los menores, sobre todo para aquellos de familias de rentas bajas, es la forma de hacer la cultura efectivamente accesible para todos. En este sentido, diseñar políticas de provisión de servicios artísticos y culturales para el desarrollo de la creatividad en el seno de las comunidades, así como en ámbitos como el hospitalario, es clave; tanto para el equilibrio emocional y la dotación de herramientas de resiliencia desde etapas tempranas como para el impulso de la industria cultural. 


\section{REFERENCIAS BIBLIOGRÁFICAS}

Adorno, T. (1970 [1983]). Teoría estética. Barcelona: Orbis.

Ateca-Amestoy, V., y Prieto-Rodriguez, J. (2013). Forecasting accuracy of behavioural mo-dels for participation in the arts. Journal of Operational Research, 229, 124-131.

Baumol, W. J., y Bowen, W. (1966). Performing Arts, The Economic Dilemma. New York: The Twentieth Century Fund.

Blaug, M. (2001). Where are we now on cultural economics. Journal of economic surveys, 15(2), 123-143.

Carpena, A. y López, O. (2012). Creatividad y emociones positivas en el alumnado con problemas de salud. International Journal of Developmental and Educational Psychology, 1(4), 37-44.

Cruz, O., Mejías, M. y Machado, Y. (2014). Caracterización emocional de niños escolares hospitalizados con enfermedades crónicas, Revista Cubana de Pediatría, 86(4), 462-469.

De Valdenebro, X. (2001). El arte y el juego, Educación y educadores, 4, 61-70.

Del Barrio, V. y Mestre, V. (1989). Evaluación psicológica en niños hospitalizados, Revista de Psicología de la Salud, 1, 83103.

Eco, U. (1970). La definición de arte. Barcelona: Ediciones Martínez Roca, S.A.

Erroteta, J. M. (2011). Estructuración del psiquismo en la temprana infancia. Esbozos del pensamiento creativo. En E. Moreno, El psicoanálisis y la capacidad creativa en el ser humano (pp.29-40). Madrid: Biblioteca Nueva.

Falk, M., y Katz-Gerro, T. (2016). Cultural participation in Europe: Can we identify common determinants? Journal of Cul- tural Economics, 40, 127-162.

Fernández, M. I. (2003). Creatividad, arte terapia y autismo. Un acercamiento a la actividad Plástica como proceso creativo en niños autistas, Arte, individuo y sociedad, 15, 135-152.

Fernández-Cao, M. L. (2011). Memoria, ausencia e identidad. El arte como terapia. Madrid: Eneida.

Fernández-Castillo, A. y López-Naranio, I. (2006). Transmisión de emociones, miedo y estrés infantil por hospitalización, International Journal of Clinical and Health Psychology, 6(3), 631-645.

Gili, M., Roca, M., Basu, S., McKee, M., y Stuckler, D. (2013). The mental health risks of economic crisis in Spain: evidence from primary care centres, 2006 and 2010. Eur. J. Public Health, 23, 103-108.

Granados, I. M. (2009). Interrelaciones entre la creatividad, el arte, la educación y la terapia, Arte y movimiento, 1, 51-62.

Gómez, L. J. (2020). Breve estudio de las medidas adoptadas durante el Estado de Alarma durante la gestión de la crisis sanitaria ocasionada por el Covid-19, GABILEX, Revista del Gabinete Jurídico de Castilla-La Mancha, 21, 19-70.

Guo, Y. R., Cao, Q. D., Hong, Z. S., Tan, Y. Y., Chen, S. D., Jin, H. J. ... y Yan, Y. (2020). The origin, transmission and clinical therapies on coronavirus disease 2019 (COVID-19) outbreak-an update on the status, Military Medical Research, 7(1), 1-10.

Gutiérrez, E. (2017). Arteterapia con familias de niños con cáncer. Dinámica familiar en la creación plástica (tesis doctoral). Universidad del País Vasco.

Hernández, E. y Rabadán, J. A. (2013). La hospitalización: un paréntesis en la vida del 
niño, Atención educativa en población infantil hospitalizada, Perspectiva educacional, 52(1), 167-181.

Lizasoáin, O. y Polaino-Lorente, A. (1992). Efectos y manifestaciones psicopatológicas de la hospitalización infantil, Revista Española de Pediatría, 48(1), 52 60.

Lizasoáin, O. y Ochoa, B. (2003). Repercusiones de la hospitalización pediátrica en el niño enfermo, Cuadernos de Ciencias médicas, 5, 75-85.

López-Naranjo, I. y Fernández-Castillo, A. (2004). Aspectos psicosociales y evolutivos de la hospitalización infantil Revista de Psicología Social Aplicada, 14(3), 5-28.

López-Naranjo, I. y Fernández-Castillo, A. (2006). Hospitalización infantil y atención psico-educativa en contextos excepcionales de aprendizaje, Revista de educación, 341, 553-577.

Lotman, Y. (1982). El arte como lenguaje. Estructura del texto artístico. Madrid: Istmo.

Mayo, E. (2018). Vulnerabilidad, ruptura social y arteterapia, Papeles de Arteterapia para la Educación y la Inclusión Social, 13, 33-52.

Monroy, M. R. (2006). Arte, creatividad y aprendizaje. La imaginación como vehículo de la movilidad interior: duelo y simbolización artística, Reencuentro, $46,1-12$.

O'Hagan, J. W. (1996). Access to and participation in the arts: the case of those with low incomes/educational attainment. Journal of cultural economics, 20(4), 269-282.

Palacios, M., Santos, E., Velázquez, M. V. y León, M. (2020). COVID-19, una emergencia de salud pública mundial. Revista Clínica Española (en prensa).

Perez-Villadoniga, M. J., y Suarez-Fernandez, S. (2019). Education, income and cultural participation across Europe. Cuadernos Económicos del ICE, 98.

Ramos, C. (2020). Covid-19: la nueva enfermedad causada por un coronavirus, Salud pública de México, 62(2), 225 227.

Rothan, H. A. y Byrareddy, S. N. (2020). The epidemiology and pathogenesis of coronavirus disease (COVID-19) outbreak, Journal of autoimmunity, 102433.

Shereen, M. A., Khan, S., Kazmi, A., Bashir, N. y Siddique, R. (2020). COVID-19 infection: origin, transmission, and characteristics of human coronaviruses, Journal of Advanced Research, 24, 91-98.

Suárez-Fernández, S., Prieto-Rodríguez, J., y Pérez-Villadoniga, M. J. (2020). The changing role of education as we move from popular to highbrow culture. Journal of Cultural Economics, 44, 189212.

Urbanos-Garrido, R. M., y Lopez-Valcarcel, B. $G$. (2015). The influence of the economic crisis on the association between unemployment and health: an empirical analysis for Spain. The European Journal of Health Economics, 16(2), 175 184

Vigotsky, L. S. (2003). La imaginación y el arte en la infancia. Madrid: Akal.

Winnicott, D. W. (1971). Realidad y juego. Barcelona: Gedisa. 
